MATEC Web of Conferences 22,01053 (2015)

DOI: $10.1051 /$ matec conf/ 20152201053

(C) Owned by the authors, published by EDP Sciences, 2015

\title{
Communication Support Technology Research and Network Design of Mobile Energy Efficiency Test and Energy-saving Assessment System
}

\author{
Ming Zhong \\ China Electric Power Research Institute, Beijing, China \\ Chongyang Du \\ North China Electric Power University, Baoding, Hebei, China \\ Songsong Chen \\ China Electric Power Research Institute, Beijing, China \\ Baoqu Yang \& Qiang Xie \\ State Grid Liaoning Electric Power Development Co., Ltd., Shenyang, Liaoning, China
}

\begin{abstract}
In order to solve the communication problems when the mobile energy efficiency and energy-saving assessment system is applied to energy monitoring, the authors introduced two schemes about building wireless network which is introduced based on the demand of the mobile energy efficiency test and energy-saving assessment system. These two schemes are based on WDS (Wireless Distribution System), WSN (Wireless Sensor Network), they effectively solved the problem that signals could not be transmitted through the net when the mobile energy efficiency test and energy-saving assessment system is used to monitor the monitoring points which are located underground or in an extensive area.
\end{abstract}

Keywords: energy efficiency monitoring; WLAN; WDS; wireless bridge

\section{INTRODUCTION}

The mobile energy efficiency test and energy-saving assessment system is a system designed for the energy efficiency monitoring, analysis and diagnosis. Its monitoring objects are industrial and commercial users. This system consists of vehicle analysis platform and energy efficiency monitoring system which deployment is flexible and reusable. This system is characterized with convenient deployment, simple operation, and accurate analysis. However, according to the operation so far, the mobile energy efficiency test and energy-saving assessment system is facing communication problems in two situations: the monitoring points located in underground buildings and the monitoring points located in an area where is extensive or there are too many obstacles. In these two situations, the signal can be hardly transmitted through the mobile energy efficiency test and energy-saving assessment system because of the topographic factors. In order to solve the problems in data transmission, there is a need to design communication networks fitted for the demands of the mobile energy efficiency test and energy-saving assessment system. In this paper, we have considered the communication needs of the mobile energy efficiency test and energy-saving assessment system, made a research on the board band, transmission delay of the system and the technology of constructing wireless network. In the end, we put forward schemes for communication network construction based on portable monitoring terminals, wireless bridges and centralization equipment for energy efficiency data.

\section{COMMUNICATION SYSTEM OF THE MO- BILE ENERGY EFFICIENCY AND ENER- GY-SAVING ASSESSMENT SYSTEM}

The mobile energy efficiency test and energy-saving assessment system consists of the automobile analysis platform and the monitoring system. The automobile analysis platform consists of the basic software platform, the power consumption simulation software of typical energy-using system, the energy efficiency assessment program, the software of energy-saving analysis model and relevant servers and network equipment. The monitoring system consists of the portable monitoring terminals, the centralization equipment for energy efficiency data and the supporting equipment such as wireless bridges and routers. The monitoring system can be deployed by workers according to the needs. The monitoring equipment connected with automobile analysis platform by centralization equipment for energy efficiency data.

The communication system hardware of the mobile energy efficiency test and energy-saving assessment system has four components: centralization equipment 
MATEC Web of Conferences

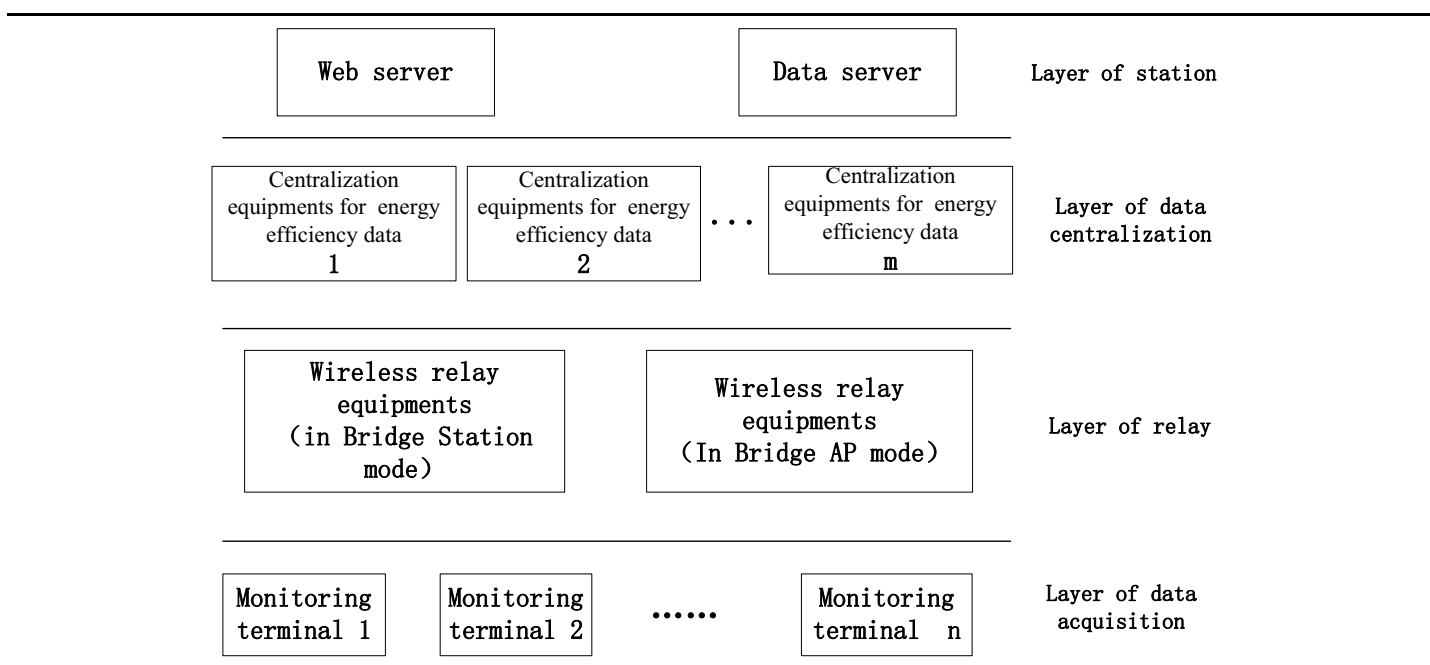

Figure 1. Construction of layers

for energy efficiency data, wireless relay equipment, portable monitoring terminals and database with its server. According to their functions, the communication system can be divided into four layers as shown in Figure 1.

As we can see from this figure, the construction of communication system of the mobile energy efficiency and energy-saving assessment system is constructed in four layers: data acquisition layer, delay layer, data centralization layer, and station layer.

In the data acquisition layer, portable monitoring terminal is the main component. The main function of portable monitoring terminals is to obtain data from monitoring points. Each portable monitoring terminal can monitor 4 types of information at the same time. They can obtain information, store them temporarily and transmit them to network. If the distance of transmission is not long enough, they can transmit it to centralization equipment for energy efficiency data directly; if the distance is too long, then they transmit to the relay equipment most nearby.

In the relay layer, its mainly component is the wireless bridge. The bridges are working in different mode to fit different situations. They have two modes: Bridge Station and Bridge AP. The main function of this layer is to ensure the data transmission between the portable monitoring terminals and centralization equipment for energy efficiency data, keep the data link in conditions such as long distance and complicated topography.

In the data centralization layer, the main component is the centralization equipment for energy efficiency data. Centralization equipment for energy efficiency data can aggregated data transmitted from portable monitoring terminals preliminarily and then transmitted data to the analysis program and database.

In the station layer, there are Web servers and Database servers. As the front interface of the system and network, its main function is to provide database ac- cess interface for background analysis software of enterprise, provide services for the invocation of monitoring data. The main functions of database server are data collection and analysis. According to the analysis above, we can conclude that the characteristics of the mobile energy efficiency test and energy-saving assessment system is a wireless communication network which is easily deployed, flexible and easy to expand.

\section{PERFORMANCE REQUIREMENTS FOR THE COMMUNICATION SYSTEM OF THE MO- BILE ENERGY EFFICIENCY AND ENER- GY-SAVING ASSESSMENT SYSTEM}

\subsection{Requirements of board band}

The acquisition of data about energy efficiency information of electric users needs enough bandwidth, including the highest bandwidth and the basic bandwidth. The packet transmission rate is different from different monitoring objects. And paper [1] has pointed out that the monitoring objects of energy efficiency acquisition are divided into classes A-F as shown in Table 1:

Table 1. Packet transmission rate of different monitoring objects

Communication Packet transmission rate of different monitoring objects in per

\begin{tabular}{lllllll} 
& \multicolumn{1}{l}{ hour } & & & & & \\
\cline { 2 - 7 } & Class & Class & Class & Class & Class & Class \\
& $\mathrm{A}$ & $\mathrm{B}$ & $\mathrm{C}$ & $\mathrm{D}$ & $\mathrm{E}$ & $\mathrm{F}$ \\
\hline The highest & 8328.6 & 8328.6 & 7540.9 & 3677.5 & 3677.5 & 7685.0 \\
\hline The basic & 1803.0 & 1803.0 & 1897.5 & 1133.6 & 1133.6 & 1517.1 \\
\hline
\end{tabular}


The monitoring object of the mobile energy efficiency test and energy-saving assessment system is of class $\mathrm{C}$ and the formula for the need of bandwidth is shown as follows:

$$
B=\frac{\text { Packet transmission rate } \times 8}{3600 \times 1000}
$$

The mobile energy efficiency and energy-saving assessment system has four centralization equipment, each of them can centralize data from 60 monitoring terminals. The monitoring terminal can monitor 4 measuring points at the same time. Based on the data mentioned above, we can calculate the bandwidth of the energy efficiency test and energy-saving assessment mobile system needs to work.

The highest is shown as follows:

$$
B_{H}=\frac{7540.9 \times 8 \times 60 \times 4}{3600 \times 1000}=4.021 \mathrm{Kbit} / \mathrm{s}
$$

The basic is shown as follows:

$$
B_{L}=\frac{1897.5 \times 8 \times 60 \times 4}{3600 \times 1000}=1.012 \mathrm{Kbit} / \mathrm{s}
$$

\subsection{Reliability requirements and delay limits}

According to the operation index given by paper [1], the requirements of delay of the mobile energy efficiency and energy-saving assessment system are shown as follows:

The signal of telemetry over limit that transmitted from monitoring to automobile platform should within $30 \mathrm{sec}$.

The telemetry situation change information transmit from monitoring to automobile platform should be within $2 \mathrm{sec}$.

Control commands transmission through the network should be within $6 \mathrm{sec}$ [2].

When the network is shut down because of some reason, it should be recovered in at least $15 \mathrm{~min}$.

\subsection{Requirements for security}

The data obtained by the mobile energy efficiency and energy-saving assessment system is about the energy efficiency of the users and it's very important. So the communication network of the system should have security mechanism to keep the attack from outside net away from the system, and make sure that information will not be leaked out [3].

In some case, the system needs to be connected to the public network. In this case, it needs to take access point name (APN), virtual private network (VPN), security isolation, access control, authentication and encryption and other security measures [4]. For the area has more danger, there is a need to establish a remote secure encrypted channel. The use of authentication, network border protection, isolation devices and other safety measures provides authentication for original data, anti-replay, data encryption, data integrity verification, and multiple levels for security. These measures can be effectively used to against the network information steal, tampering with network data, network replay attacks, and ensure that the encryption of transmission data of power can be used to ensure data security and prevent attacks from the internal network [5].

\section{SCHEMES OF COMMUNICATION NETWORK CONSTRUCTION}

\subsection{Scheme against monitoring points located un- derground}

\subsubsection{Situation}

When the monitoring points are located underground where the signals can hardly reach, the data transmits well between the monitoring terminal and the platform. Wired connection can solve transmission problem well, but its deployment is complicated and cumbersome. This way can hardly be used to match the "simple and flexible" character of the mobile energy efficiency test and energy-saving assessment system. Most deployment of wires on site which is temporary needs to be applied to the enterprise where the monitoring points located in, and it is time-consuming. The wires that lying temporarily are with poor protection and can be easily damaged by the cars and passengers.

\subsubsection{Specific design of scheme}

In this situation, the communication network of the mobile energy efficiency test and energy-saving assessment system consists of two parts: WSN and WIFI. WSN and WIFI are connected by wireless bridges as shown in

Figure 2:

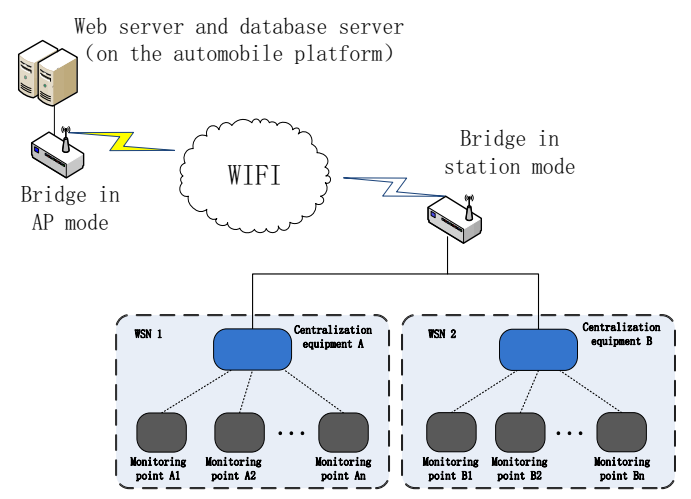

Figure 2. Network topology diagram 
WSN consists of portable monitoring terminal and centralization equipment for energy efficiency data. Each portable terminal is responsible for monitoring the energy efficiency of measurement point; each measurement point comprises a number of monitoring points. These monitoring points may be physical parameters, such as pressure, flow rate, may also be electrical parameters, such as voltage, current, inductance, and so on. Each portable monitoring terminal is connected to equipment for energy efficiency data centralization and the centralization equipment for energy efficiency data which is connected to a bridge that worked under Bridge Station mode [6].

In WSN networks, portable monitoring terminals and centralization equipment for energy efficiency data connected in star structure. The star structure is easy for network management and operations. When the monitoring strategy changes, we can add or delete portable monitoring terminals by the centralization equipment for energy efficiency data at any time and do not cause a greater impact on the network topology.

Centralization equipment for energy efficiency data has a dual-mode communication function. On one hand, it can be connected to software analysis platform of energy-saving company by GPRS wireless public network; on the other hand, it can be connected to mobile platform by WIFI network. The way of connection between centralization equipment for energy efficiency data and automobile platform depends on the distance between them [7].

(1) When the distance is short, the centralization equipment for energy efficiency data can be connected to automobile platform by wired network card.

(2) When the distance is too long to connect directly, the centralization equipment for energy efficiency data is connected to a bridge work in Bridge Station mode in a wired way. Bridge Station is one of the working modes of bridge. When a bridge is working in this mode, it will create a client interface on WLAN module. Equipment which is connected to this client interface can visit another bridge working in AP mode which located near automobile platform and connect to the switch on the platform in wired way [8].

After the switch of platform building connection with the centralization equipment for energy efficiency data, the platform can receive the data from monitoring terminal and transmit to the database server. Database server analyzes the data and obtains the analysis results. Monitoring software will analyze the energy efficiency of the enterprise. The automobile analysis platform can obtain the energy efficiency information and the analysis results through web server [9].

\subsection{Scheme against monitoring points located in extensive area}

\subsubsection{Situation}

In some situations, the area which is needed to be monitored is too extensive or there are too many obstacles that make the effective communication distance be shorter than usual. The maximum wireless effective communication distance of portable monitoring terminal is $100 \mathrm{~m}$, so when the system is in a situation as described above, the data transmission between portable monitoring terminal and centralization equipment for energy efficiency data will hardly be ensured.

\subsubsection{Specific design of scheme}

In order to solve the problem which is faced in extensive monitoring area, the scheme will use the bridges with WDS function as the relay equipment of the mobile energy efficiency test and energy-saving assessment system [10]. WDS, namely the Wireless Distribution System, allows wireless devices bridged by electromagnetic waves, the wireless signal can be expanded and extended in this system. This system can make wireless network coverage broader than usual and can greatly improving the stability and efficiency of wireless networks [11]. WDS function can solve the coverage shortage problems of single-site monitoring device. Working in wireless way can greatly reduce the complexity of the deployment and well fit the need of the mobile energy efficiency test and energy-saving assessment system. By bridges with WDS function, we can construct the relay network as shown in

Figure 3 [12]:

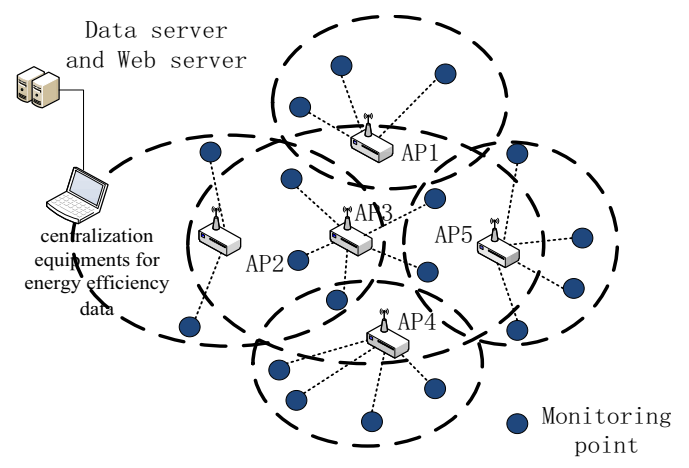

Figure 3. Network topology diagram

Bridges in Figure 3 are working in AP mode. Each bridge can build up a smaller WSN with the monitoring points under its coverage. By using the same SSID and encryption, the WSN can enlarge its coverage [13].

In the above case, AP1 has three monitoring points in its coverage and its SSID is W1. Without expanding, W1 only has three points and the centralization 
equipment for energy efficiency data is beyond its signal coverage, the monitoring points could not transmit the energy efficiency information back to centralization equipment for energy efficiency data and automobile platform. However, if all AP in this figure are opening the WDS function and using the same SSID W1 and same encryption, then the WSN $\mathrm{W} 1$ can cover all the monitoring points in the figure and the centralization equipment for energy efficiency data is under the signal coverage [14].

At the receiving end of the monitoring network, on one hand, the centralization equipment for energy efficiency data is connected to the switch on the automobile platform; on the other hand, it is connected to the AP most nearby though WIFI and by this way, it can be connected to the monitoring network. By the $5 \mathrm{AP}$ in this figure, we achieved the data transmission from monitoring points to the centralization equipment, and solved the transmission problem exist in condition with extensive monitoring area.

\section{CONCLUSION}

In this paper, we researched the problems that the mobile energy efficiency test and energy-saving assessment system faced in the actual use. In order to solve the problems in situation that monitoring points located underground and located in extensive area, we designed two schemes of wireless network construction. By using the technology of wireless bridge and WLAN, we successfully design the structure of wireless network on site and solved the problem of information transmission.

\section{REFERENCES}

[1] Ziyan Zhao \& Hao Hu. 2011. A calculation method based on intelligent business section of the distribution of electricity communication network traffic. Power System Technology, (11).

[2] AKYOL B A., KIRKHAM H \& CLEMENTS S L. 2010 A survey of wireless communication for the electric power system.

[3] CLARK A. \& PAVLOVSKI C J.2010. Wireless networks for the smart energy grid: application aware networks.

[4] Jinping Cao, Jianming Liu. \& Xiangzhen Li.2013. Technology Solutions of Wireless Network for Smart Distribution Networks. Automation of Electric Power Systems. (37)

[5] J. S. Vardakas,M. K. Sidiropoulos,M. \& D. Logothetis.2008. Performance behavior of IEEE 802.11 distributed coordination function. IET Circuits, Devices \& Systems.

[6] Jiaxing Wang.2014. Application of Wireless LAN. Electronics and Software Engineering, (22).

[7] Yuan Zhou, Zezhou Luo \& Hongcheng Zhuang. 2013. sensor-assisted coverage self-optimization for wireless local area network. Proceedings of 2013 22nd Wireless and Optical Communication Conference.

[8] Yuntao Fan, Hewu Li \& Wenqi Sun.2014. Modeling AP's Substitutability in Dense-deployed WLANs. Proceedings of 2014 IEEE International Conference on Progress in Informatics and Computing.

[9] Yuhong Zhu \& Changqing Xu.2008. Model of distribution system indoor based on wireless LAN. Computer Engineering. (07).

[10]Qunsong Huo 2010. Research about WLAN Networking and Data Forwarding Mode. Peking Posts and Telecommunications University.

[11]Linjia Yin 2010. Analysis and research about WLAN wireless local area network performance in noisy environment. Peking Posts and Telecommunications University.

[12]Lei Li, Baoxian Zhang, Heqing Huang \& Haitao Liu.2010. Research on wireless sensor network path coverage. Electronics \& Information Technology. (10)

[13]Zhenhua Bi.2013. Video surveillance systems based on wireless bridge technology and its applications. Chinese Academy of Sciences University.

[14]Qiang Xu. 2013. Design and Implementation of video surveillance system audio and video synchronization technology. Hangzhou Electronic Science and Technology University. 